\title{
RELACIÓN ENTRE CONCENTRACIÓN SANGUÍNEA DE $\beta$-HIDROXIBUTIRATO E INDICADORES PRODUCTIVOS, REPRODUCTIVOS Y DE SALUD EN HATOS JERSEY Y HOLSTEIN
}

\author{
Alejandro Saborío-Montero ${ }^{1 / *}$, Jorge Ml. Sánchez ${ }^{* \dagger}$ \\ Palabras clave: $\beta$-hidroxibutirato; cetosis; producción de leche; reproducción. \\ Keywords: $\beta$-hydroxybutyrate; ketosis; milk yield; reproduction.
}

Recibido: 23/09/15 Aceptado: 03/12/15

\begin{abstract}
RESUMEN
Se correlacionó la concentración sanguínea de $\beta$-hidroxibutirato ( $\beta \mathrm{HBA}$ ) con indicadores productivos, reproductivos y de salud, en 2 hatos de vacas de alta producción, de las razas Jersey y Holstein, ubicados en Oreamuno (Jersey) y La Unión (Holstein) de Cartago, Costa Rica; ambos con vacas primíparas y multíparas, en sistemas de pastoreo. Se tomaron muestras de sangre de vasos coccígeos de 117 y 114 vacas Jersey a los $8 \pm 3$ y $30 \pm 3$ días de lactancia (DL), y de 72 y 50 Holstein a $5 \pm 3$ y $30 \pm 3$ DL, respectivamente. En cada muestra se analizó in situ la concentración de $\beta$ HBA, y la información productiva, reproductiva y de salud del hato se registró en el programa VAMPP Bovino 3.0. Mediante análisis de asociación, se establecieron relaciones entre concentración de $\beta$ HBA e indicadores de interés. Un análisis de razón de oportunidades ("odds ratio") señaló a las Jersey que sufrieron hipocalcemia clínica con 3,63 veces la probabilidad de tener concentraciones de $\beta$ HBA superiores a 1,4 mmol. $1^{-1}$, en contraste con las sanas. Sin embargo, la información obtenida sugiere que concentraciones promedio de $\beta \mathrm{HBA}$ de 0,66 (IC 95\%: $0,59-0,74)$ a los $8 \pm 3$ DL en vacas Jersey y de 0,59 (IC 95\%: 0,52-0,65) a los 5 \pm 3 DL en las vacas Holstein, así como concentraciones promedio de
\end{abstract}

1 Autor para correspondencia. Correo electrónico: alejandro.saboriomontero@ucr.ac.cr
ABSTRACT

Blood concentration of $\beta$-hydroxybutyrate in Jersey and Holstein herds under grazing and its association with productive, reproductive and health indicators. The blood concentration of $\beta$-hydroxybutyrate ( $\beta \mathrm{HBA}$ ) in 2 high yielding cow herds, one of Jersey breed and the other Holstein, was correlated with productive, reproductive and health indicators. The Jersey herd was grazing in Oreamuno and the Holstein in La Union, both in Cartago, Costa Rica, and including primiparous and multiparous cows. Blood samples were taken from coccygeal vessels of 117 and 114 Jersey cows at $8 \pm 3$ and $30 \pm 3$ days of lactation (DL), and from 72 and 50 Holstein cows at $5 \pm 3$ and $30 \pm 3$ DL, respectively; concentration of $\beta \mathrm{HBA}$ was analyzed in situ and the herd productive, reproductive and health information was recorded in the VAMPP Bovino 3.0. Association analyses done, to establish relationships between $\beta$ HBA concentration and indexes of interest, indicated that Jersey animals suffering clinical hypocalcemia had 3.63 times a chance to have $\beta$ HBA concentrations

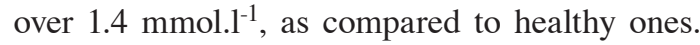
However, the results suggest that average $\beta \mathrm{HBA}$ concentrations of 0.66 (95\% CI: 0.59 to 0.74 ) at $8 \pm 3 \mathrm{DL}$ in Jersey cows and of $0.59(95 \% \mathrm{CI}$ :

Universidad de Costa Rica, Centro de Investigación en Nutrición Animal y Escuela de Zootecnia, Costa Rica. 
$\beta$ HBA de 0,91 (IC 95\%: 0,78-1,05) en las Jersey y de 0,69 (IC 95\%: 0,58-0,80) en las Holstein a los $30 \pm 3$ DL no afectaron la producción láctea ni la reproducción en hatos cuya alimentación se basó en uso intensivo de forrajes tropicales más 2,5 a $3 \mathrm{~kg}$ por vaca por día de un alimento balanceado (1,9 Mcal EN $\mathrm{E}_{\mathrm{L}} \cdot \mathrm{kg}^{-1}, 48 \%$ almidón).

\section{INTRODUCCIÓN}

Las vacas lecheras por lo general reducen el consumo de materia seca (Grummer 1993, Hayirli et ál. 2002) y pierden condición corporal (Saborío y Sánchez 2014) durante las 3 semanas previas al parto. Asímismo, sus requerimientos energéticos para producir leche se incrementan rápidamente al inicio de la lactancia lo que causa un balance energético negativo importante durante esta fase de su ciclo productivo, llamada también periodo de transición. Para compensar este desbalance, el sistema homeostático promueve la movilización de las reservas de grasa, produciéndose ácidos grasos no esterificados también llamados NEFA's por sus siglas en inglés (Non Esterified Fatty Acids) como producto de la disociación de triglicéridos mediante el proceso de lipólisis (Drackley 1999). Estos NEFA's bajo condiciones normales son metabolizados en el hígado para satisfacer las necesidades de energía de los procesos fisiológicos involucrados en el final de la gestación, inicios de la lactancia y reproducción (Butler et ál. 2006, Roche et ál. 2009).

Cuando hay acumulación de ácidos grasos en el hígado antes del parto, o bien cuando la movilización de las reservas de grasa en forma de NEFA's al inicio de la lactancia sobrepasa la capacidad del hígado para oxidarlos y generar energía, se produce el $\beta$ HBA que al acumularse
$0,52-0.65)$ at $5 \pm 3 \mathrm{DL}$ in Holsteins, as well as $\beta \mathrm{HBA}$ average concentrations of $0.91(95 \% \mathrm{CI}$ : 0.78 to 1.05 ) for Jerseys and 0.69 (95\% CI: 0.58 to 0.80 ) for Holsteins at $30 \pm 3$ DL, did not affect milk production nor reproduction in dairy herds whose feeding was based on intensive use of tropical forages, plus a balanced food at a rate of 2.5 to $3 \mathrm{~kg}$ per cow per day $\left(1.9 \mathrm{Mcal} \mathrm{EN}_{\mathrm{L}} \cdot \mathrm{kg}^{-1}\right.$, $48 \%$ starch).

en el organismo causa el desbalance en el metabolismo energético denominado cetosis. Diferentes autores (Baird 1982, NRC 2001) han documentado que este desbalance metabólico produce una disminución sustancial en la producción de leche, causada por una reducción rápida en la generación de energía en el organismo. Además, en las vacas de alta producción puede haber infiltración de triglicéridos en los hepatocitos al inicio de la lactancia, aspecto que hace el hígado graso (Hocquette y Bauchart 1999).

Según sea la concentración sanguínea de $\beta \mathrm{HBA}$, la cetosis puede clasificarse como clínica o subclínica. La cetosis en su forma subclínica ha sido definida como la concentración sanguínea de $\beta$ HBA entre 1,4 y 2,9 mmol. ${ }^{-1}$ (Carrier et ál. 2004, Oetzel 2007, Duffield et ál. 2009). Desde la década de los 90's, ésta es la enfermedad metabólica de mayor prevalencia en los Estados Unidos de América, ya que sobrepasa a la acidosis ruminal y a la fiebre de leche, atrayendo la atención de investigadores (Oetzel 2007). La incidencia de cetosis clínica en sistemas de producción de leche estabulados oscila entre 2 a $15 \%$ en vacas recién paridas (Duffield 2000), mientras que la incidencia de la forma subclínica de la enfermedad ha sido estimada entre 40 y $60 \%$ (Emery et ál. 1964, Simensen et ál. 1990, Duffield et ál. 1998). Diferentes autores han asociado la cetosis subclínica con la producción de leche y el desempeño reproductivo en hatos lecheros (Bobe et ál. 2004, 
Duffield et ál. 2009, Gillund et ál. 2001, Gustafsson et ál. 1993, Santschl et ál. 2011, Vickers et ál. 2013, Walsh et ál. 2007). La mayoría de estos autores concuerdan en que la cetosis afecta negativamente la producción de leche y la eficiencia reproductiva; sin embargo la mayoría de estos estudios ha sido realizada en hatos de sistemas confinados propios de zonas de clima templado.

En los países ubicados en zonas tropicales la producción de leche se basa en el uso intensivo de los pastos $\mathrm{C}_{4}$, los cuales tienen cantidades de energía neta de lactancia que oscilan entre 1,0 y 1,35 Mcal.kg ${ }^{-1}$ de MS (3X) (Sánchez 2007), cantidades inferiores a las que tienen las gramíneas $\mathrm{C}_{3}$ (1,5 Mcal.kg-1 de MS, 3X) (NRC 2001). Lo anterior marca diferencias en la alimentación y nutrición energética de ambas poblaciones de ganado bovino y puede incidir sobre la prevalencia de cetosis. En general, en las últimas décadas el potencial para la producción de leche en los hatos tropicales ha aumentado en forma significativa y las vacas Jersey se han convertido en una alternativa atractiva para los productores debido a su tamaño, capacidad de adaptación y calidad de la leche. Esta última característica le da valor agregado a la misma, pero a su vez incrementa las necesidades de energía de los animales. De igual manera la raza Holstein aún es la raza más utilizada para lechería especializada en Costa Rica. El objetivo de esta investigación fue explorar la asociación entre la concentración sanguínea de $\beta \mathrm{HBA}$ en sangre en un hato comercial de vacas Jersey a los $8 \pm 3$ y $30 \pm 3$ DL y un hato comercial de vacas Holstein a los $5 \pm 3$ y $30 \pm 3$ DL, ambos en pastoreo, con parámetros productivos, reproductivos y de salud. Lo anterior puede contribuir a crear estrategias entre técnicos y productores sobre el manejo de la nutrición energética y ampliar la información referente a este desbalance metabólico.

\section{MATERIALES Y MÉTODOS}

Un total de 117 vacas de un hato lechero comercial de la raza Jersey y 72 vacas de un hato comercial lechero de la raza Holstein, ubicados en Santa Rosa de Oreamuno, Cartago, Costa Rica ( $9^{\circ} 55^{\prime}$ Latitud Norte, $83^{\circ} 51^{\prime}$ Longitud Oeste, 2350 msnm) y San Ramón de Tres Ríos, La Unión, Cartago, Costa Rica (0957' Latitud Norte, $83^{\circ} 58^{\prime}$ Longitud Oeste, $1650 \mathrm{msnm}$ ) respectivamente, fueron objeto de estudio desde agosto de 2012 hasta agosto de 2014. Se realizaron visitas semanales a las fincas durante el periodo de estudio con el objetivo de medir in situ la concentración de $\beta$ HBA sanguíneo durante las 2 primeras semanas posparto $(5 \pm 3$ o $8 \pm 3 \mathrm{DL})$ y durante la cuarta semana de lactancia $(30 \pm 3$ DL). La dieta previa al parto (-21 días al parto) de ambos hatos se basó en pastoreo intensivo de kikuyo (Kikuyuocloa clandestina) y $4 \mathrm{~kg}$ de alimento concentrado bajo en calcio (16\% PC, $1,7 \mathrm{Mcal}$ de $\mathrm{EN}_{\mathrm{L}} / \mathrm{kg}, 35 \%$ almidón, $0,2 \% \mathrm{Ca}$ ) por vaca por día, mientras que la dieta de vaca lactante consistió en el pastoreo de kikuyo y 6 a $8 \mathrm{~kg}$ de alimento balanceado (16\% PC, 1,9 Mcal EN $\mathrm{EN}_{\mathrm{L}}$ ' $\mathrm{kg}, 48 \%$ almidón, $0,9 \% \mathrm{Ca}$ ) para las vacas Jersey y 8 a $10 \mathrm{~kg}$ del mismo alimento balanceado para las vacas Holstein.

Las muestras de sangre fueron extraídas de los vasos coccígeos de 117 vacas Jersey (24\% primíparas, $76 \%$ multíparas, número de parto promedio $=3,35$ ) a los $8 \pm 3$ días de lactancia y 114 vacas Jersey (24\% primíparas, $76 \%$ multíparas, número de parto promedio $=3,32$ ) a los $30 \pm 3$ DL. Tres vacas no pudieron ser muestreadas debido a mortalidad (no asociada a cetosis) o descarte del hato. Las muestras de sangre de las vacas Holstein fueron tomadas de la misma manera a 72 hembras ( $42 \%$ primíparas, $58 \%$ multíparas, número de parto promedio $=2,61$ ) y 50 hembras (42\% primíparas, $58 \%$ multíparas, número de parto promedio $=2,64$ ) a los $5 \pm 3$ y $30 \pm 3$ DL, respectivamente. De las vacas Holstein 22 no pudieron ser muestreadas en el periodo $30 \pm 3 \mathrm{DL}$ por motivos de mortalidad, descarte del hato, pérdida de arete identificador y coordinación fuera del alcance de los investigadores. Se calificó la condición corporal de los animales semanalmente en una escala de 1 a 5 puntos mediante la metodología descrita por Ferguson et ál. (1994). 
Cada muestra fue analizada in situ para determinar la concentración de $\beta$ HBA. Para esto se utilizó un dispositivo electroquímico (Optium ${ }^{\circledR}$ Xceed, Abbott Diabetes Care ${ }^{\circledR}$ ) cuya sensibilidad (96\%) y especificidad (97\%) han sido previamente reportadas (Iwersen et ál. 2009). Valores umbrales de 1,4 a 2,9 mmol. $\mathrm{l}^{-1}$ de $\beta \mathrm{HBA}$ sanguíneo y $>2,9$ mmol. $\mathrm{l}^{-1}$ fueron utilizados para definir los animales positivos a cetosis subclínica y clínica, respectivamente. La producción de leche y la información reproductiva se registró quincenalmente a través del programa VAMPP ${ }^{\circledR}$ (Veterinary Automated Management and Production Control Program). La unidad experimental, fue cada una de las vacas y se utilizó la razón de oportunidades (Odds Ratio) para definir el grado de asociación entre la concentración de $\beta$ HBA sanguíneo y la ocurrencia de hipocalcemia clínica, donde las vacas se agruparon en sanas o en enfermas, según la manifestación de síntomas clínicos de hipocalcemia clínica durante el periparto, y en los grupos $<1,4$ mmol. $\mathrm{l}^{-1}$ y $\geq 1,4$ mmol. $1^{-1}$ de $\beta$ HBA sanguíneo. Se utilizó la prueba $t$ de Student para muestras independientes para comparar los índices de desempeño productivo y reproductivo entre animales con algún grado de cetosis y animales sanos. Para comparar la concentración de este cuerpo cetónico en diferentes periodos de una misma raza se utilizó la prueba $\mathrm{t}$ de Student para muestras pareadas. La información fue analizada con los programas Infostat $1,0^{\circledR}$ (Di Rienzo et ál. 2011) y SPSS ${ }^{\circledR}$ versión 20. Las diferencias significativas fueron declaradas cuando $\mathrm{p}<0,05$ o el intervalo de confianza al $95 \%$ del OR calculado no incluyó el valor 1.

\section{RESULTADOS Y DISCUSIÓN}

\section{Asociación entre la concentración de $\beta H B A$ sanguíneo e índices de desempeño productivo y reproductivo}

Durante el periodo de la investigación, el hato Jersey en estudio mostró producciones corregidas a $305 \mathrm{~d}(\approx 6000 \mathrm{~kg})$ mayores a los valores promedio de la raza Jersey en el hato nacional $(\approx 5150 \mathrm{~kg})$ para animales nacidos en 2008 y 2009 , de igual manera, el hato Holstein fue más productivo $(\approx 8000 \mathrm{~kg})$ que la media del hato nacional $(\approx 7000 \mathrm{~kg})$ para la raza en animales comparables. Los valores promedio para la raza se obtuvieron según datos del Programa de Evaluación Genética de Ganado Lechero de Costa Rica (PCVET-EMV 2015).

En el hato Jersey, la producción de leche ni el comportamiento reproductivo de las vacas multíparas difirió $(p>0,05)$ entre aquellas con cetosis y aquellas que no tuvieron este desbalance metabólico (Cuadro 1). En este mismo hato se han reportado (Saborío y Sánchez 2013) valores de incidencia de 3,51 y $9,65 \%$ de cetosis a los $30 \pm 3$ DL (tipo I), en sus formas clínica y subclínica, respectivamente. Asimismo, la incidencia de cetosis subclínica a los $8 \pm 3$ d (tipo II) fue $4,27 \%$, sin presencia de cetosis clínica, durante este periodo. 
Cuadro 1. Índices de desempeño productivo y reproductivo en vacas multíparas ${ }^{1}$ en pastoreo de la raza Jersey, con o sin cetosis por periodo de muestreo.

\begin{tabular}{|c|c|c|c|c|}
\hline $\begin{array}{l}\text { Periodo de } \\
\text { muestreo }\end{array}$ & Índice de desempeño & Estatus de cetosis ${ }^{2}$ & Media (DE) & Valor $\mathrm{P}^{3}$ \\
\hline \multirow{5}{*}{$8 \pm 3 \mathrm{DL}$} & Producción total de leche (kg) & $\begin{array}{l}- \\
+\end{array}$ & $\begin{array}{l}6331,2(1342,3) \\
5734,8(761,7)\end{array}$ & 0,382 \\
\hline & Producción de leche a 305d (kg) & $\begin{array}{l}- \\
+\end{array}$ & $\begin{array}{l}6261,5(1025,7) \\
5718,8(765,8)\end{array}$ & 0,301 \\
\hline & Periodo abierto (días) & $\begin{array}{l}- \\
+\end{array}$ & $\begin{array}{r}115,5(47,4) \\
90,1(39,1)\end{array}$ & 0,363 \\
\hline & Servicios por concepción & $\begin{array}{l}- \\
+\end{array}$ & $\begin{array}{l}2,2(1,1) \\
1,7(1,2)\end{array}$ & 0,453 \\
\hline & Intervalo entre partos (días) & $\begin{array}{l}- \\
+\end{array}$ & $\begin{array}{l}400,3(57,1) \\
370,7(45,4)\end{array}$ & 0,380 \\
\hline \multirow{5}{*}{$30 \pm 3 \mathrm{DL}$} & Producción total de leche (kg) & $\begin{array}{l}- \\
+\end{array}$ & $\begin{array}{l}6231,7(1366,3) \\
6192,6(1301,8)\end{array}$ & 0,922 \\
\hline & Producción de leche a 305d (kg) & $\begin{array}{l}- \\
+\end{array}$ & $\begin{array}{l}6136,1(1069,3) \\
6335,9(1005.5)\end{array}$ & 0,550 \\
\hline & Periodo abierto (días) & $\begin{array}{l}- \\
+\end{array}$ & $\begin{array}{l}114,7(50,5) \\
109,2(26,7)\end{array}$ & 0,609 \\
\hline & Servicios por concepción & $\begin{array}{l}- \\
+\end{array}$ & $\begin{array}{l}2,1(1,1) \\
2,5(1,0)\end{array}$ & 0,279 \\
\hline & Intervalo entre partos (días) & $\begin{array}{l}- \\
+\end{array}$ & $\begin{array}{l}401,0(61,1) \\
385,7(28,5)\end{array}$ & 0,441 \\
\hline
\end{tabular}

1 Se tabularon únicamente la información de las vacas multíparas debido a que ninguna hembra primípara presentó valores superiores a 1,4 mmol. $1^{-1}$ en el periodo $8 \pm 3 \mathrm{~d}$ y solo una hembra primípara presentó cetosis subclínica en el periodo $30 \pm 3$ d por lo que no fue posible obtener medidas de tendencia central ni de variabilidad para ésta agrupación.

2 Estatus de cetosis (+) significa que la concentración de $\beta$ HBA sanguíneo fue $\geq 1,4 \mathrm{mmol.1}^{-1}$. El símbolo (-) implica que la concentración de $\beta$ HBA sanguíneo fue $<1,4$ mmol.1 $1^{-1}$. Las medias de concentración de $\beta$ HBA sanguíneo para (+) y (-) fueron 1,90 y 0,63 para $8 \pm 3$ DL y 2,46 y 0,76 para $30 \pm 3$ DL en las vacas multíparas.

3 Se compararon las medias de los índices de desempeño mediante la prueba t de student para muestras independientes. 
En el hato Holstein no se presentó ningún caso clínico y la prevalencia subclínica de la enfermedad para el periodo $5 \pm 3$ y $30 \pm 3$ DL fue 1,4 y $4,0 \%$, respectivamente. En este hato no se consideró apropiado clasificar a los animales diagnosticados como positivos en un grupo puesto que fueron únicamente un animal para el periodo $5 \pm 3$ DL y 2 animales para el periodo $30 \pm 3$ DL. El limitado número de observaciones en el grupo positivo, impide realizar comparaciones objetivas entre medias de indicadores productivos y reproductivos.

Los resultados en el hato Jersey difieren de los obtenidos en otros estudios, en los que se ha encontrado una disminución en la producción de leche y en el desempeño reproductivo de los animales debido a la incidencia de cetosis subclínica durante el primer mes de la lactancia (Duffield et ál. 2009, Gillund et ál. 2001, Gustafsson et ál. 1993, Fourichon et ál. 2003, Vickers et ál. 2013, Walsh et ál. 2007). Lo anterior podría deberse a que los estudios citados se han realizado en vacas de la raza Holstein con producciones corregidas a 305 d superiores a $8000 \mathrm{~kg}$ y por lo tanto con demandas energéticas mayores a los animales Jersey estudiados, cuyas producciones de leche corregida a 305 d fueron cercanas a los 6000 $\mathrm{kg}$. Sin embargo se debe valorar que el análisis realizado en esta investigación tiene un número limitado de animales y que la falta de significancia estadística podría estar afectada por el tamaño de muestra.

La prevalencia de la enfermedad en el hato Holstein, tanto en su forma clínica como subclínica, fue inferior a la reportada en otros estudios. Oetzel (2007) menciona valores cercanos a $15 \%$ para cetosis clínica en hatos Holstein estabulados y Duffield (2000) reporta proporciones superiores al $40 \%$ para la forma subclínica del padecimiento en hatos de la misma raza bajo estabulación. Una posible causa asociada a la menor prevalencia de la enfermedad encontrada en este hato podría ser la baja condición corporal con la que parieron los animales, la pérdida de condición corporal para el hato Holstein durante la lactancia fue inferior a 0,25 puntos (Figura 1) que pasó de 3,18 puntos al momento del parto, hasta 2,99 puntos a la quinta semana de lactancia.

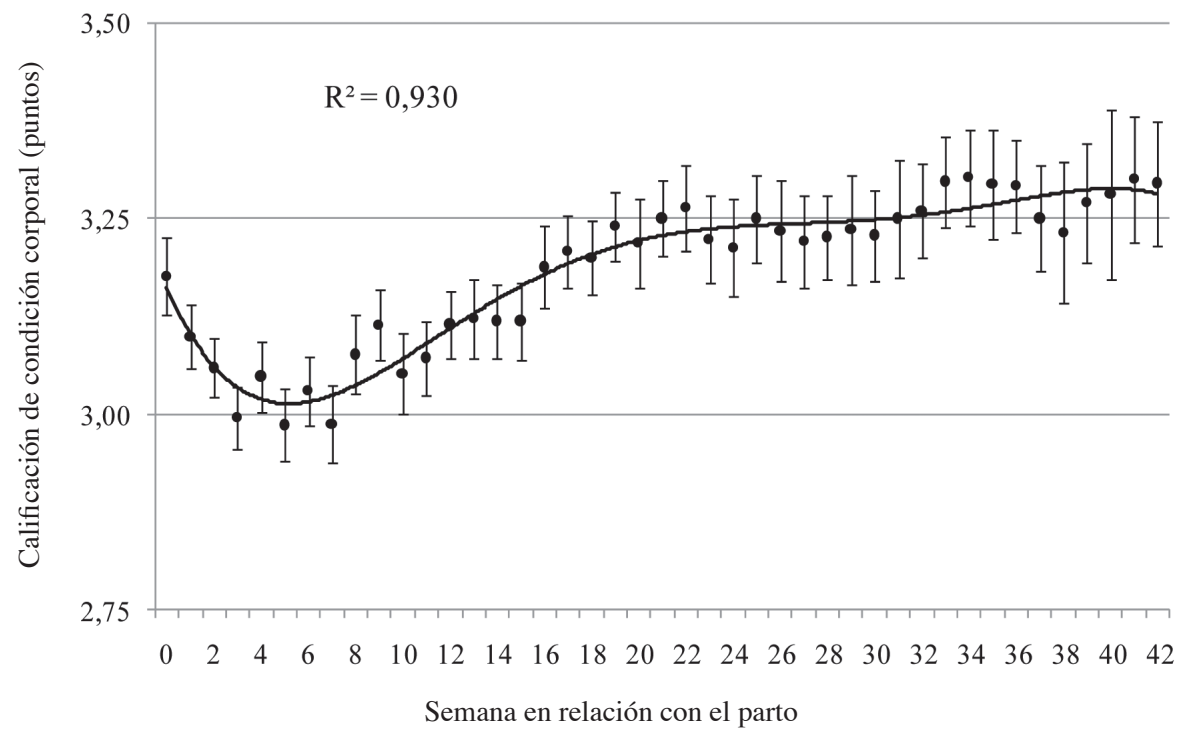

Fig. 1. Variación de la condición corporal durante la lactancia de un hato Holstein en pastoreo ubicado en La Unión de Cartago. 
En el hato Jersey los valores promedio de $\beta$ HBA sanguíneo fueron relativamente bajos a los $8 \pm 3 \mathrm{DL}$, con valores de $1,90 \mathrm{mmol}^{-1} \mathrm{l}^{-1}$ (IC 95\%: 1,39-2,41) para los animales diagnosticados con algún grado de cetosis y $0,61 \mathrm{mmol}^{-\mathrm{l}^{-1}}$ (IC 95\%: $0,55-0,67)$ para los que no sufrieron ningún grado de la enfermedad. En el periodo $30 \pm 3$ DL dichas concentraciones aumentaron a $2,41 \mathrm{mmol}^{-1} \mathrm{l}^{-1}$ (IC 95\%: $1,89-2,93$ ) y $0,69 \mathrm{mmol}^{-1}$ (IC 95\%: $0,63-$ $0,75)$ en promedio para animales positivos $\mathrm{y}$ negativos a cetosis, respectivamente.

Los animales Jersey tuvieron concentraciones promedio de $\beta$ HBA de 0,67 (IC 95\%: 0,59$0,74)$ y $0,91 \mathrm{mmol}^{-1}$ (IC 95\%: $\left.0,78-1,05\right)$ a los $8 \pm 3$ DL y $30 \pm 3$ DL, las vacas Holstein tuvieron concentraciones sanguíneas de $\beta \mathrm{HBA}$ de 0,59 (IC 95\%: $0,52-0,65$ ) y $0,69 \mathrm{mmol}^{-1}$ (IC 95\%: $0,58-$ $0,80)$ a los $5 \pm 3$ DL y $30 \pm 3$ DL, respectivamente
(Figura 2). Las concentraciones de $\beta \mathrm{HBA}$ a los $30 \pm 3$ DL fueron mayores $(\mathrm{p}<0,01)$ para las vacas Jersey que para las Holstein. Esta mayor concentración de $\beta \mathrm{HBA}$ en el periodo $30 \pm 3$ DL podría deberse a una mayor movilización de reservas corporales para la raza Jersey en relación con la raza Holstein. Una mayor movilización de grasa subcutánea ocurre en animales con mayor condición corporal al momento del parto (Saborío y Sánchez 2014). En el caso de los hatos en estudio, el hato Jersey parió con un promedio de 3,87 puntos de condición corporal, mientras que el hato Holstein presentó una condición corporal promedio de 3,18 puntos. El cambio en condición corporal promedio desde la semana del parto hasta el punto más bajo de la curva para el hato Jersey y Holstein fue 0,65 y 0,19 puntos, respectivamente.

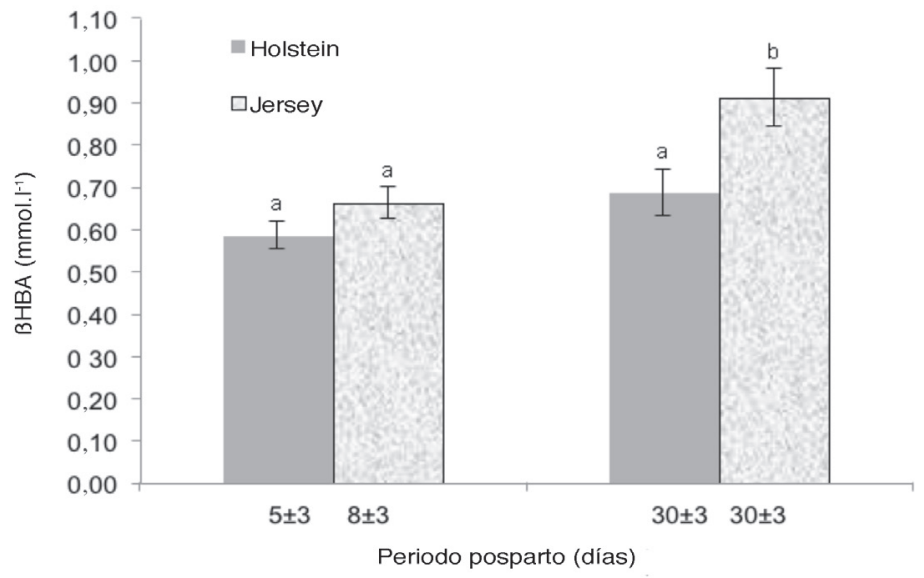

Fig. 2. Concentración de $\beta$ HBA según periodo de muestreo posparto, en vacas Jersey y Holstein en pastoreo.

* Barras con letras distintas implican diferencias altamente significativas $(\mathrm{p}<0,01)$.

\section{Asociación entre concentración sanguínea de BHBA e incidencia de fiebre de leche}

Una agrupación de animales Jersey según presentación de fiebre de leche al parto mostró que $29 \%$ de las vacas que tuvieron fiebre de leche, presentaron concentraciones sanguíneas superiores a 1,4 mmol. $^{-1}$ de $\beta$ HBA a los $30 \pm 3$ DL, en contraposición solamente $10 \%$ de las vacas que no presentaron el padecimiento al parto tuvieron concentraciones de $\beta$ HBA superiores a 1,4 mmol. $\mathrm{l}^{-1}$ en el mismo periodo. Estos resultados coinciden con hallazgos previamente reportados en la literatura en la cual se describen interrelaciones entre las enfermedades metabólicas (Curtis et ál. 1983, Erb y Grohn 1988, Grohn et ál. 1989, Goff y Horst 1997). En el caso del 
hato Holstein, no se encontró relación alguna entre las concentraciones de $\beta \mathrm{HBA}$ con esta enfermedad, sin embargo esto podría deberse a que la raza Jersey es la más susceptible a fiebre de leche (Saborío et ál. 2015).

Un análisis de la razón de oportunidades (odds ratio) como medida de asociación indicó que los animales Jersey que presentaron fiebre de leche tuvieron 3,63 (IC 95\%: 1,10-11,91) veces la probabilidad de tener concentraciones $\geq 1,4$ mmol. $1^{-1}$ de $\beta$ HBA en sangre a los $30 \pm 3$ DL en relación con los animales que no tuvieron fiebre de leche (Cuadro 2).

Cuadro 2. Concentración de $\beta$ HBA sanguíneo $\left(m m o l .1^{-1}\right)$ a los $30 \pm 3$ DL según la presencia o no de fiebre de leche durante el periparto en 114 vacas de la raza Jersey en pastoreo.

\begin{tabular}{|c|c|c|c|c|c|c|}
\hline & \multicolumn{2}{|c|}{$\beta \mathrm{HBA}<1,4\left(\mathrm{mmol} . \mathrm{l}^{-1}\right)$} & \multicolumn{2}{|c|}{$\beta \mathrm{HBA} \geq 1,4\left(\mathrm{mmol} . \mathrm{l}^{-1}\right)$} & \multirow[t]{2}{*}{$\mathrm{OR}^{*}$} & \multirow[t]{2}{*}{ IC $95 \%$} \\
\hline & $\mathrm{n}$ & $(\%)$ & $\mathrm{n}$ & $(\%)$ & & \\
\hline Sin fiebre de leche & 87 & 90 & 10 & 10 & 1 & \\
\hline Con fiebre de leche & 12 & 71 & 5 & 29 & 3,63 & $1,10-11,91$ \\
\hline
\end{tabular}

*OR="Odds Ratio" =Razón de oportunidades =Razón de productos cruzados.

Estos resultados indican que la probabilidad de sufrir algún grado de cetosis es significativamente mayor para los animales que sufrieron fiebre de leche durante el periparto. Sin embargo podría existir un efecto subyacente del número de partos en esta asociación, ya que el incremento en el número de partos, además de haber sido relacionado previamente al aumento en la incidencia de cetosis, también ha sido asociado a una diminución en las concentraciones sanguíneas de calcio durante el periparto y por consiguiente al aumento en la incidencia de fiebre de leche (Reinhardt et ál. 2011, Sánchez y Saborío 2014a, Sánchez y Saborío 2014b). Algunas de las razones propuestas para explicar la disminución en la concentración sanguínea de calcio conforme aumenta el número de parto incluyen una mayor actividad osteoclástica en animales en crecimiento $\left(1^{\circ}\right.$ y $2^{\circ}$ lactancia) que les permite una mejor respuesta del metabolismo del calcio. Otra posible razón es la menor producción de calostro en éstos animales, lo que disminuye las demandas de este mineral al inicio de la lactancia (NRC 2001). Además el número de receptores intestinales de vitamina $\mathrm{D}$ (esenciales en el metabolismo del calcio) es menor conforme se incrementa la edad de las vacas (Horst et ál. 1990).

En ganado lechero es frecuente que durante el periodo de transición (Parto \pm 21 días), varias enfermedades metabólicas se presenten a la vez en un mismo animal (Curtis et ál. 1983).

Los resultados de la investigación muestran que aunque las vacas Jersey multíparas tuvieron mayor concentración sanguínea de $\beta \mathrm{HBA}$, estos animales no presentaron afectación significativa de indicadores de desempeño productivo ni reproductivo. De igual forma la baja prevalencia de cetosis y las bajas concentraciones de $\beta \mathrm{HBA}$ en el hato Holstein indican que hay que realizar más investigación en esta línea para determinar la verdadera afectación de los hatos costarricenses.

\section{CONCLUSIONES}

Bajo las condiciones del estudio y según los resultados de los análisis de registros, se considera que no existe evidencia para asegurar que la cetosis incide sobre los rendimientos productivos o reproductivos de los animales afectados. Sin embargo es necesaria más investigación con 
tamaños de muestra mayores para aseverar que esta enfermedad no afecta dichos rendimientos bajo condiciones tropicales en hatos pastoriles a nivel poblacional. A pesar del hallazgo de una asociación estadística entre algún grado de cetosis y la incidencia de fiebre de leche, se considera que esta asociación podría obedecer a la relación de ambas enfermedades con el número de partos de los animales afectados, condición que genera un efecto confuso. Se recomienda establecer un programa de diagnóstico preciso para cetosis en la lactación temprana para animales sintomáticos, mediante el uso de medidores cuantitativos electroquímicos de $\beta$ HBA sanguíneo, para controlar acertadamente la prevalencia de la enfermedad en los hatos comerciales.

\section{AGRADECIMIENTOS}

Los autores expresan su gratitud al Ing. Agr. José Joaquín Jiménez Zamora propietario de Hacienda Pasquí, a Don Roberto y su hija Denise Gurdián por facilitar los hatos para realizar este estudio. También el agradecimiento al Sr. Jorge Enrique Volio Elbrech y Med. Vet. Carlos Luis Madriz Bonilla por la recopilación de la información productiva y reproductiva de Hacienda Pasquí y a los funcionarios de ambas lecherías. Gracias al personal del programa CRIPAS, (Centro Regional de Investigación para la Producción Animal Sostenible) por permitirnos utilizar el software VAMPP Bovino 3,0.

\section{LITERATURA CITADA}

BAIRD D.G. 1982. Primary ketosis in the high producing dairy cow: clinical and subclinical disorders, treatment, prevention and outlook. J. Dairy Sci. 65:1-10.

BOBE G., YOUNG J.W., BELTZ D.C. 2004. Invited review: pathology, etiology, prevention, and treatment of fatty liver in dairy cows. J. Dairy Sci. 87:3105-3124.

BUTLER S.T., PELTON S.H., BUTLER. W.R. 2006. Energy balance, metabolic status, and the first postpartum ovarian follicle wave in cows administere propylene glycol. J. Dairy Sci. 89:2938-2951.

CARRIER J., STEWART S., GODDEN S., FETROW J., RAPNICKI P. 2004. Evaluation and use of three cowside test for detection of subclinical ketosis in early postpartum cows. J. Dairy Sci. 87:3725-3735.

CURTIS C.R., ERB H.N., SNIFFEN G.J., SMITH R.D., POWERS P.A., SMITH M.C., WHITE M.E., HILLMAN R.B., PEARSON E.J. 1983. Association of parturient hypocalcemia with eight periparturient disorders in Holstein cows. J. Am. Vet. Med. Assos. 183(5):559-561.

DI RIENZO J.A., CASANOVES F., BALZARINI M.G., GONZALEZ L., TABLADA M., ROBLEDO C.W. 2011. InfoStat versión 2011. Grupo InfoStat, FCA, Universidad Nacional de Córdoba, Argentina. URL http://www.infostat.com.ar

DRACKLEY J.K. 1999. Biology of dairy cows during the transition period: The final frontier? J. Dairy Sci. 82:2259-2273.

DUFFIELD T.F. 2000. Subclinical ketosis in lactating dairy cattle. Vet Clin. North Am. Food Anim. Pract. 16:231-253.

DUFFIELD T.F., LISSEMORE K.D., MCBRIDE B.W., LESLIE K.E. 2009. Impact of hyperketonemia in early lactation dairy cows on health and production. J. Dairy Sci. 92:571-580.

DUFFIELD T.F., SANDALS D., LESLIE K.E., LISSEMORE K., MCBRIDEB.W., LUMSDENJ.H., DICK P., BAGG R. 1998. Efficacy of monensin for prevention of subclinical ketosis in lactating dairy cows. J. Dairy Sci. 81:2866-2873.

EMERY R.S., BURG N., BROWN L.D., BLANK G.N. 1964. Detection, occurrence, and prophylactic treatment of borderline ketosis with propylene glycol feeding. J. Dairy Sci. 47:1074-1079.

ERB H.N., GRÖHN Y.T. 1988. Epidemiology of metabolic disorders in the periparturient dairy cow. J. Dairy Sci. 71(9):2557-2571.

FERGUSON J.D., GALLIGAN D.T., THOSEN N. 1994. Principal descriptors of body condition score in Holstein cows. J. Dairy Sci. 72:2695-2703.

FOURICHON C., SEEGERS H., MALHER X. 2003. Quantification of the effects of ketosis on reproduction in dairy cows by meta-analysis. $11^{\text {th }}$ ICPD Proceedings. Abstracts, p. 317.

GILLUND P., REKSEN O., GRÖHN Y.T., KARLBERG K. 2001. Body condition related to ketosis and reproductive performance in Norwegian dairy cows. J. Dairy Sci. 84:1390-1396.

GOFF J.P., HORST R.L. 1997. Physiological changes at parturition and their relationship to metabolic disorders. J. Dairy Sci. 80:1260-1268.

GRÖHN Y.T., ERB H.N., McCULLOCH C.E., SALONIEMI H.S. 1989. Epidemiology of metabolic disorders in dairy cattle: Associations among host characteristics, disease and production. J. Dairy Sci. 72:1876-1885.

GRUMMER R.R. 1993. Etiology of lipid-related metabolic disorders in periparturient dairy cows. J. Dairy Sci. 76:3882-3896. 
GUSTAFSSON A.H., ANDERSSON L., EMANUELSON U. 1993. Effect of hyperketonaemia, feeding frequency and intake of concentrate and energy on milk yield in dairy cows. Animal Production 56:51-60.

HAYIRLI A., GRUMMER R.R., NORDHEIM E.V., CRUMP P.M. 2002. Animal and dietary factors affecting feed intake during the prefresh transition period in Holstein. J. Dairy Sci. 85:3430-3443.

HOCQUETTE J.F., BAUCHART D. 1999. Intestinal absorption, blood transport and hepatic and muscle metabolism of fatty acids in preruminant and ruminant animals. Reprod. Nutr. Dev. 39:27-48.

HORST R.L., GOFF J.P., REINHARDT T.A. 1990. Advancing age results in reduction of intestinal and bone 1, 25 dyhidroxyvitamin D receptor. Endocrinology 126:1053-1057.

IWERSEN M., FALKENBERG U., VOIGTSBERGER R., FORDERUNG D., HEUWIESER W. 2009. Evaluation of an electronic cowside test to detect subclinical ketosis in dairy cows. J. Dairy Sci. 92:2618-2624.

NATIONAL RESEARCH COUNCIL. 2001. Nutrient Requirements of Dairy Cattle. 7th rev. ed. National Academy Press. Washington, D.C., pp. 184-213.

OETZEL G. 2007. Herd-level ketosis-diagnosis and risk factors. American Association of Bovine Practitioners 40th Annual Conference. September 19. Vancouver, BC, Canada, pp. 67-91.

PCVET-EMV. 2015. Evaluación Genética de Ganado lechero - Costa Rica. Cronograma de evaluaciones. Tendencias. Consultado el 5-02-2015. Disponible en http://www.medvet.una.ac.cr/posgrado/gen/tenden. html

REINHARDT T.A., LIPPOLIS J.D., MCCLUSKEY B.J., GOFF J.P., HORST R. L. 2011. Prevalence of subclinical hypocalcemia in dairy herds. The Veterinary Journal 188(1):122-124.

ROCHE J.R., FRIGGENSN.C., KAY J.K., FISHER M.W., STAFFORD K.J., BERRY D.P. 2009. Invited Review: Body condition score and its association with dairy cow productivity, health, and welfare. J. Dairy Sci. 92:5769-5801.

SABORÍO A., SÁNCHEZ J. 2013. Prevalencia y factores de riesgo relacionados con la cetosis clínica y subclínica tipo I y II en un hato de vacas Jersey en Costa Rica. Agronomía Costarricense 37(2):17-29.
SABORÍO A., SÁNCHEZ J. 2014. Evaluación de la condición corporal en un hato de vacas Jersey en pastoreo en la zona alta de Cartago. Variaciones durante el ciclo productivo. Agronomía Costarricense 38(1):55-65.

SABORÍO A., VARGAS B., ROMERO J.J., SÁNCHEZ J. 2015. Risk factors associated with milk fever occurrence in Costa Rican dairy cattle. (Abstract). Animal Health. Lactating Cows, pp. 319-320. T15. J. Anim. Sci Vol. 93, Suppl. s3 Print ISSN: 0021-8812. Online ISSN: 1525-3163. / J. Dairy Sci. Vol. 98, Suppl. 2. Print ISSN: 0022-0302. Online ISSN 15253198. Available in: http://www.jtmtg.org/JAM/2015/ abstracts/315.pdf

SÁNCHEZ J. 2007. Utilización eficiente de las pasturas tropicales en la alimentación del ganado lechero, pp. 14-30. In: R. Tejos et ál. (eds). Manejo y utilización de pastos y forrajes en sistemas de producción animal. Barquisimeto, Venezuela.

SÁNCHEZ J., SABORÍO A. 2014a. Prevalencia de hipocalcemia en cuatro hatos Jersey en pastoreo en Costa Rica. Agronomía Costarricense 38(2):33-41.

SÁNCHEZ J., SABORÍO A. 2014b. Hipocalcemia e hipomagnesemia en un hato de vacas Holstein, Jersey y Guernsey en pastoreo. Agronomía Costarricense 38(2):55-65.

SANTSCHL D.E., LEFEBVRE D.M., CUE R.I., GLRARD C.L., PELLERLN. 2011. Incidence of metabolic disorders and reproductive performance following a short (35-d) or conventional (60-d) dry period management in commercial Holstein herds. J. Dairy Sci. 94:3322-3330.

SIMENSEN E., HALSE K., GILLUND P.1990. Ketosis treatment and milk yield in dairy cows related to milk acetoacetate levels. Acta Vet. Scand. 31(4):433 440.

VICKERS L.A., WEARY D.M., VEIRA D.M., VON KEYSERLINGK M.A.G. 2013. Feeding a higher forage diet prepartum decreases incidences of subclinical ketosis in transition dairy cows. J. Anim. Sci. 91:886-894.

WALSH R.B., WALTON J.S., KELTON D.F., LEBLANC S.J., LESLIE K.E., DUFFIELD T.F. 2007. The effect of subclinical ketosis in early lactation on reproductive performance of postpartum dairy cows. J. Dairy Sci. 90:2788-2796. 